\title{
Stellar populations with ELTs
}

\author{
Rosemary F.G. Wyse ${ }^{1}$ and Gerard Gilmore ${ }^{2}$ \\ ${ }^{1}$ Department of Physics and Astronomy, Johns Hopkins University, Baltimore, \\ MD 21218, USA \\ email: wyse@pha.jhu.edu \\ ${ }^{2}$ Institute of Astronomy, Madingley Rd, Cambridge CB3 0HA, UK \\ email: gil@ast.cam.ac.uk
}

\begin{abstract}
The star formation, mass assembly and chemical enrichment histories of galaxies, and their present distributions of dark matter, remain encoded in their stellar populations. Distinguishing the actual distribution functions of stellar age, metallicity and kinematics at several locations in a range of galaxies, sampling across Hubble types and representative environments, is the information required for a robust description of galaxy histories. Achieving this requires large aperture, to provide the sensitivity to reach a range of environs and Hubble types beyond the Local Group, to provide high spatial resolution, since the fields are crowded, and preferably with optical performance since age-sensitivity is greatest near the main-sequence turn-off, and metallicity-sensitivity for these warm stars is greatest in the optical.
\end{abstract}

Keywords. Galaxies: stellar content, formation; Stars: populations; Telescopes

\section{Understanding galaxy formation: the context}

\subsection{The theory}

The working paradigm for cosmological structure formation is gravitational instability of initially low-amplitude, adiabatic, Gaussian and near scale-invariant density fluctuations in a universe dominated by cold dark matter (with dark energy accelerating the expansion of the Universe at late times). The first structures that form are small, perhaps equal in mass to a dwarf galaxy now, and large galaxies result from the hierarchical merging and accretion of many small systems. The merging history of a typical massive-galaxy dark halo is fairly straightforward to calculate, since only gravity is involved. However, most simulations lack the resolution to model the smallest scales with more than a few particles, and cannot follow how far inside a 'parent' halo a merging satellite penetrates, crucial to determine the effect on the baryonic galaxy. The state-of-the-art Millenium Simulation (Springel et al. 2005) has a particle mass of $8.6 \times 10^{8} h^{-1} \mathrm{M}_{\odot}$ and a resolution of $5 h^{-1} \mathrm{kpc}$. The incorporation of baryonic physics - in particular gas dissipation, star formation and feedback - is much more complex, and the results much more model dependent. Feedback from an active nucleus is only very recently beind incorporated into the simulations (e.g. Croton et al. 2006), with the establishment of the correlations between black hole properties and stellar bulge/spheroid (e.g. Gebhardt et al. 2000; Ferrarese \& Merritt 2000) and recognition of their probably fundamental nature (e.g. Silk \& Rees 1998; Kauffmann \& Haenelt 2000). This of course adds more uncertain physics and associated parameters into the models.

Abadi et al. (2003) present a recent high-resolution simulation of the formation of a present-day disk galaxy that demonstrates many of the important aspects, including the 
outstanding problem of how to include star formation and gas physics. Generic predictions for disk galaxies include the following:

- Extended disks form late, after a redshift of unity, or a lookback time of $\sim 8$ Gyr, in order to avoid losing too much angular momentum during active merging at earlier times

- A large disk galaxy should have hundreds of surviving satellite dark haloes at the present day

- The stellar halo is formed from disrupted satellites

- Minor mergers (a mass ratio of $\sim 10-20 \%$ between the satellite and the disk - a much smaller ratio between the satellite and the larger host dark halo) into a disk heat it, forming a thick disk out of a pre-existing thin stellar disk, and create torques that drive gas into the central (bulge?) regions

- More significant mergers transform a disk galaxy into an S0 or even an elliptical

- Subsequent accretion of gas can reform a thin disk

- Stars can be accreted into the thin disk from suitably massive satellites (dynamical friction must be efficient) and if to masquerade as stars formed in the thin disk, must be on suitable high angular momentum, prograde orbits

Elliticals form by 'major mergers', with a mass ratio of approximately unity; there is much uncertainty (freedom in the models) about the gas fraction of the merging entities, and how much star formation and 'feedback' occurs during the merger (e.g. Larson \& Tinsley 1979; Zurek, Quinn \& Salmon, 1988; Kauffmann 1996; Cole, Lacey, Baugh \& Frenk 2000).

Dwarf galaxies have the most fragile global potential wells, and are expected to be the most strongly affected, in any model, by internal feedback processes, by external ionization and/or ram pressure stripping (e.g. Sandage 1965; Saito 1979; Wyse \& Silk 1985; Dekel \& Silk 1986; Efstathiou 1992; Bullock, Kravtsov \& Weinberg 2000; Robertson et al. 2005). Detailed baryonic astrophysics has been appealed to, to solve the wellestablished predicted excess of satellite halos in CDM models, compared to observed satellite galaxies (Moore et al. 1999; Klypin et al. 1999).

\subsection{Observational tests, Integrated light}

A first step in an observational approach to understanding the physics behind galaxy formation is to obtain and then analyse large datasets, to identify patterns and correlations as a means to underlying physical causal connections. The dataset could be simple images of galaxies, and the pattern the Hubble Sequence. More detailed correlations such as the various projections of the Fundamental Plane of ellipticals (Dressler et al. 1987; Djorgovski \& Davis 1987) and the Tully-Fisher relationship for spirals and associated dark matter scaling relationships (e.g. Bell \& de Jong 2001) provide more insight. The small scatter seen in these correlations at low redshift means that late random merging of stellar galaxies cannot be the dominant evolutionary trend. Indeed, correlations of the properties of the stellar populations with overall potential well depth suggest that if mergers are indeed the dominant mechanism by which galaxies form and evolve, the mergers must be gas rich, and most stars form during the mergers, with the last significant merger dominating the star formation history.

Spectroscopic surveys of faint galaxies, combined with morphological information from high-resolution HST images, have allowed investigations of the evolution of the Fundamental Plane with redshift, out to $z \sim 1$, for both cluster E/SO galaxies (e.g. van Dokkum et al. 1998) and field E/SO galaxies (e.g. Treu et al. 2005). The conclusions (Treu et al. 2005) are that the most massive systems have evolved passively, consistent with the bulk of star formation at higher redshifts, $z \sim 2$, and even for lower masses, most stars formed 
at these high redshift, but a significant fraction (up to $\sim 40 \%$ ) of stars in these systems could have formed at lower redshifts. The trend is more pronounced in clusters, as expected from hierarchical clustering, but the density dependence is weaker than model predictions. Such 'downsizing' in star formation (cf. Cowie et al. 1996) is not a natural prediction of hierarchical clustering models. The dominance of early star formation in massive systems is consistent with the results of surveys of faint galaxies selected in the near infra-red, which found a significant population of red, massive galaxies at redshifts of $z \lesssim 2$ (Glazebrook et al. 2005; Cimatti et al. 2005). That major merging does not play a major role in determining the star formation rate of galaxies over cosmic time is also the conclusion of a survey of galaxies at redshift $\sim 0.7$, combining Spitzer Space Telescope mid-IR observations (to determine the star formation rate) with morphological information from HST imaging (Bell et al. 2005). The robust identification of progenitor/descendant populations, and untangling number density evolution from luminosity evolution, requires that surveys be carried out at low, intermediate and high redshift.

Dissipationless ('dry' in current jargon) merging of ellipticals to form more massive ellipticals appears to occur in dense clusters (Tran et al. 2005), and in the field (van Dokkum 2005). The relative motions of galaxies in clusters are large compared to the depth of the internal potential well of most galaxies, leading to the expectation that only the most massive systems should merge (the merging cross-section is a steeply decreasing function of the ratio of relative velocity to internal velocity dispersion; Makino \& Hut 1997). 'Selective' merging whereby only massive systems form more massive systems may preserve the fundamental plane at least in its structural projections (Boylan-Kolchin, Ma \& Quataert 2005); chemical abundances may be a more difficult aspect.

Trends with metallicity provide strong constraints on merging, both dissipational and dissipationless. Kauffmann \& Charlot (1998) argue that generically in CDM models large ellipticals form from a few large disk galaxy progenitors (rather than a large number of small progenitors) and predict a mass-metallicity relation for ellipticals, provided there is significant star formation and strong feedback during mergers (see also Nagashima et al. 2005); this last point is the crucial one, since they found that a different star formation/feedback prescription failed to produce a mass-metallicity trend in agreement with the observations. Indeed Larson \& Tinsley (1979) demonstrated that the massmetallicity relationship for ellipticals could be reproduced in a merger scenario provided mergers were gas rich, and the efficiency of star formation increased with total mass. Another prediction, that stars in more massive ellipticals should have a younger mean age than stars in lower luminosity ellipticals does not appear to be in agreement with the papers discussed above, or with the inferred star formation histories as a function of mass from the large Sloan Digital Sky Survey (SDSS) spectroscopic database (Jimenez et al. 2005) which show the opposite trend (see Fig. 1 here). However, these spectra are for the integrated light of the central regions only, and the analysis depends on detailed comparisons with spectral synthesis models, necessarily involving inherent degeneracies among age distribution, metallicity distribution and dust extinction. An ELT with capabilities to obtain spatially resolved spectra plus deep colour-magnitude diagrams from resolved stellar populations will provide much superior capabilities to determine age and metallicity distributions. For the nearer galaxies, spectra of individual stars will provide even more power.

Kauffmann \& Charlot (1998) further predict that the mass-metallicity relationship for ellipticals should remain 'virtually unchanged out to high redshift'. With an ELT, we can test this prediction.

The suggestions above that the gas fraction and amount of star formation in a merger may vary with mass, with the most massive systems forming in dissipationless mergers, 

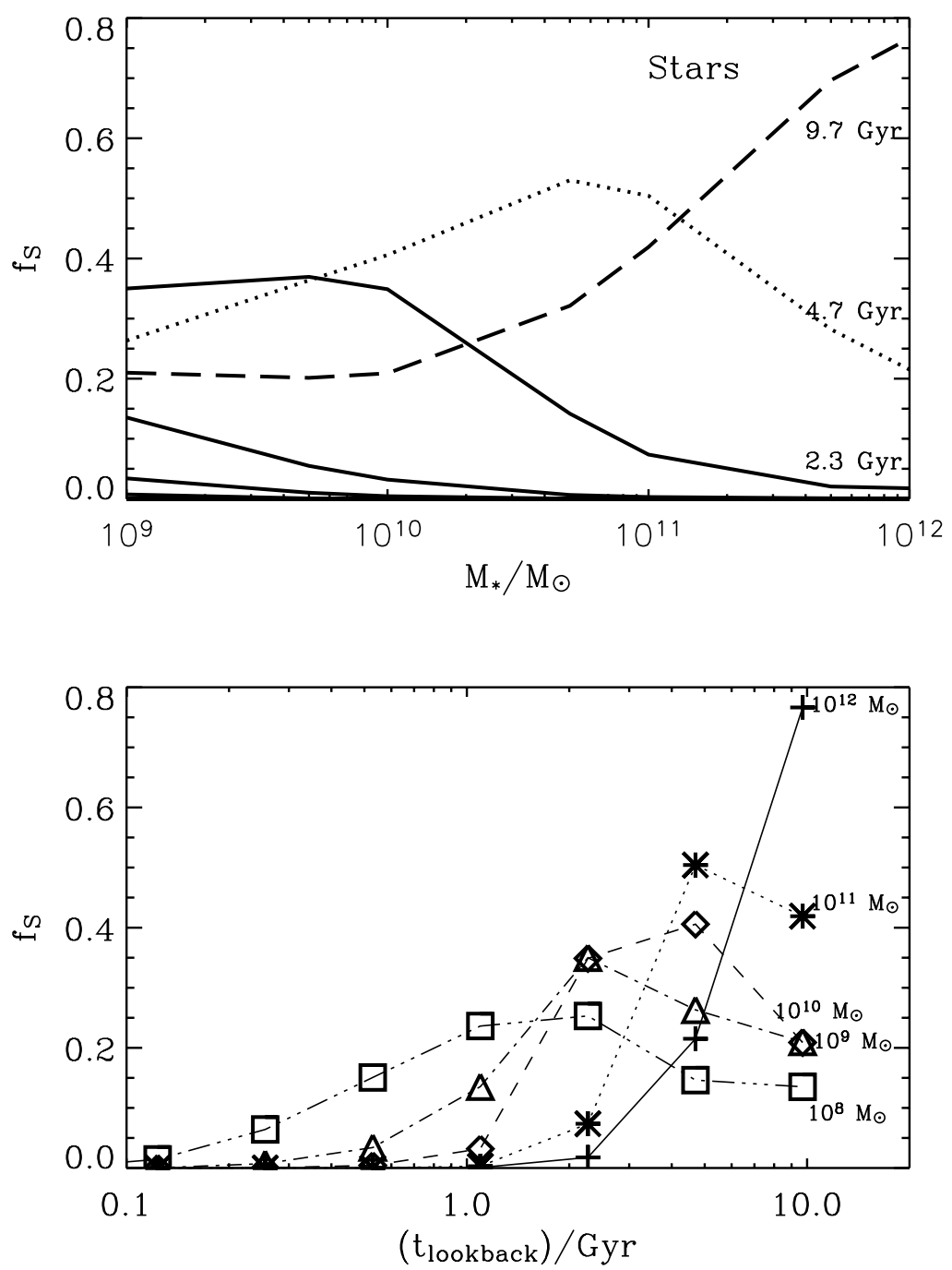

Figure 1. Taken from Jiminez et al. 2005, see also Heavens et al. 2004. Top panel: Star Formation Histories as a function of stellar mass, plotted as fraction of stellar mass formed in each of 6 age bins, only 3 labelled. Bottom panel: same thing but plotted as stellar mass bins. An ELT will enable the derivation of more detailed star formation histories, with fewer uncertainties.

should leave signatures in the mass-metallicity relationship. The correlation between central velocity dispersion and the magnesium index Mgb does show a hint of a flattening at the highest values of the velocity dispersion, as expected if simple stellar mergers with no chemical evolution occur, but there are few galaxies to define the sample. The early-type galaxies in the Sloan Digital Sky Survey do not have spectra of high enough signal-to-noise for robust measurement of line indices for each galaxy (Bernardi et al. 2003). Stacking of spectra of galaxies of similar velocity dispersion (and environment) does not show evidence of a turnover (Bernardi et al. 2003), especially when allowance is made at the highest velocity dispersions for possible superpositions of galaxies in the one 
fiber (Bernardi et al. 2005). However, spatially resolved spectra are required to establish the reality, or otherwise, of interloper companion galaxies.

Tremonti et al. (2004) studied star-forming galaxies in the SDSS spectroscopic database, confirming a strong correlation between present-day gas metallicity and stellar mass, with a suggestion of a turnover for masses greater than a few times $10^{10} \mathrm{M}_{\odot}$. This is interpreted in terms of a varying importance of gas outflows rather than anything to do with mergers.

Thus analyses of the integrated light (or, in some cases just the central regions) together with HST-resolution structural information of local and moderate redshift galaxies have pointed to a general 'anti-hierarchical' picture whereby large galaxies form their stars early, and probably assembled their mass early also.

Extended disks have been identifed by imaging in the rest-frame optical domain out to redshift $\lesssim 3$ (Trujillo et al. 2006 ), and their sizes are consistent with little evolution since then, significantly less than predicted by semi-analytic CDM models (Mo, Mao \& White 1998), but consistent with the simplest picture of gaseous infall and star formation within a fixed potential well, with the star formation rate higher in the central disk. Indeed, the interpretation from these high redshift observations is that 'stellar disks form from early on, in large haloes' (Trujillo et al. 2006).

The lowest mass galaxies can be studied in the Local Group, where the metallicityluminosity relation is extremely well-defined (see e.g. Dekel \& Woo 2003). However, this is not a straightforward metallicity-mass relation, since many of the gas-poor dwarfs apparently have little variation in total mass, with the internal kinematics pointing to a total (dark) mass of around $10^{7} \mathrm{M}_{\odot}$ (e.g. Mateo 1998, his Fig. 9). This peaked mass function is not at all expected in CDM; note that while one can change the luminosity function significantly (see Cooray \& Cen 2005) by appealing to various kinds of feedback, the mass function is not so amenable to such modifications.

\section{Resolved disk galaxies: the four(?) stellar population types}

Much more astrophysics can be derived from studies of individual stars. The large galaxies of the Local Group are accessible to study with current telescopes, and indeed the first significant spectroscopic studies of significant samples of stars in M31 and M33 are underway (e.g. Ferguson et al. 2006 and references therein; Reitzel, Guhathakurta \& Rich 2004), complemented by wide-area shallow imaging (Ibata et al. 2001; Ferguson et al. 2002) and deep narrow-field imaging with the Hubble Space Telescope (Brown et al. 2003; Ferguson et al. 2005; Brown et al. 2006). The fascinating results from these observations there is significant substructure in all stellar components, in all their properties - underline the need for large samples of stars with good spectra, for metallicities and kinematics, over as much of the face of the galaxy as possible, with matched deep colour-magnitude data.

This is what we desire from an ELT, for as broad a range of Hubble types as possible.

Such datasets will exist in the near future for the Milky Way, as a results of the new efforts to map its stellar content (e.g. RAVE, SDSS-II/SEGUE, surveys with AAOmega, WFMOS...). We already have sufficient knowledge to use the Milky Way as a template disk galaxy, and identify four stellar populations with properties that constrain the star formation history, the chemical evolution history (flows, feedback..) and the mass assembly history. There may even be a fifth type, Pop III, as yet undetected but suspected. Comparing and contrasting external galaxies with the Milky Way then constrains these crucial aspects of galaxy formation and evolution. 
- The thin disk, also known as Baade's population I. This is composed of stars and gas on high angular momentum orbits, moving about the center with close to the circular velocity, and thus with only low amplitude random motions. Such a cold thin system probably formed by dissipational collapse of gas, in a potential that is at most adiabatically changing, and conserved angular momentum to spin-up as it collapsed (see Fall \& Efstathiou 1980; Mo, Mao \& White 1998). Hierarchical merging models however predict significant angular momentum transport and produce generically disks that are too small (Navarro \& Steinmetz 1997). Appeal to 'feedback' can prevent much of the angular momentum losses from the proto-disk, but at the expense of delaying the collapse to centrifugal equilibrium (e.g. Eke, Efstathiou \& Wright 2000) and thus predicting few old stars in disks, and no extended high-redshift disks. Contributions from accretion of stellar systems into the thin disk plane is possible, and predicted in some models (e.g. Abadi et al. 2003). Identification of such substructure is complicated by the fact that dynamical perturbations certainly exist in the form of spiral arms and giant molecular cloud complexes, and create 'moving groups' (e.g. Famaey et al. 2005). However, disruption of a satellite is a viable explanation for the apparent 'ring' of stars seen in the very outer regions of the Galactic disk, if it is real (e.g. Newberg et al. 2002; Ibata et al. 2003). Perturbations to the thin disk cannot be too strong however, or the disk will be destroyed (e.g. Ostriker 1990) and thus the properties of stars in the thin disk constrains merging histories and other energetic dynamical processes. The age and metallicity distributions of the disk, well-defined only at the solar neighbourhood, point to extended infall of metal-poor gas, and steady star formation from a redshift of $\gtrsim 1.5$ (e.g. Binney et al. 2000) to the present.

- The thick disk - this was identified as a separate component some 25 years ago (Gilmore \& Reid 1980). The dominant population is old, as old as the globular cluster 47 Tuc, $\lesssim 12$ Gyr, and of intermediate metallicity in the mean, $[\mathrm{Fe} / \mathrm{H}] \sim-0.6$, with a significant spread. The chemical enrichment history revealed by the pattern of element ratios is distinct from that of stars in the thin disk (Bensby et al. 2004). A plausible origin for the thick disk is the heating of a pre-existing thin disk by a violent dynamical event such as a minor merger; the old mean age for the thick disk limits such events to have occurred only long ago, an important constraint - and a problem, if found to be a typical result - for CDM models. Thick disks are now observed in resolved stars in other galaxies (e.g. Mould 2005; Yoachim \& Dalcanton 2005) but their properties remain to be robustly determined. An ELT would allow such detailed studies beyond the Local Group.

- The central bulge - this too was not in the classic Baade list of stellar populations. The dominant stellar population in the bulge of the Milky Way is old and metal-rich, with a broad spread in metallicities. Elemental abundances are available for remarkably few stars, given the capabilities of current telescopes, and point to a fairly rapid enrichment, being dominated by products of Type II supernovae. This, together with the old age and high (phase-space) density, point to in situ formation, in a 'starburst', at high redshift. Could this be connected to the formation of the supermassive black hole at the Galactic Center? The relationships between the 'bulge', the 'bar' and the inner disk remain unclear.

- The stellar halo, also known as Baade's Population II. This is a dominantly old and metal-poor component, with Type II dominated element ratios, indicating a short duration of star formation in each of the star-forming entities that created the halo. The outer parts show indications of significant accretion, most dramatically due to the Sagittarius dwarf (Ibata, Gilmore \& Irwin 1994; Majewski et al. 2003), which is mostly intermediate-age and more metal-rich. Accretion to the dominant Population II halo can 
only have occured at early times (Unavane, Wyse \& Gilmore 1996). The Population II halo may be connected to the stellar bulge; one can tie gas outflow from halo star-forming regions, required to provide the low mean metallicity, to gas inflow to the central regions to form the bulge. The low angular momentum of proto-halo material means that it will only come into centrifugal equilibrium after collapsing in radius by a significant factor. The predicted mass ratio of bulge to halo is around a factor of ten, just as would be expected, and the specific angular momentum distributions of stellar halo and bulge match (Wyse \& Gilmore 1992; see Figure 2 here). We have yet to obtain the data to allow a study in detail of the bulge-stellar halo connection in external galaxies; an ELT would allow this.

- Population III - which we take to mean stars formed from primordial gas, most probably precursors to galaxy formation. Where are the low-mass Pop III stars? Ongoing searches for extremely low metallicity stars in the Galactic halo have not found any strong indications of a separate population (e.g. Beers et al. 2005), but have identified a few stars with extreme deficiencies in iron, and relatively strong carbon (e.g. Aoki et al. 2005). The origins of this abundance pattern are unclear. There is little observational evidence in favour of significant variations in the stellar IMF for any of the components discussed above, but there is strong theoretical prejudice that primordial stars form with a narrow range of masses, around $\sim 200 \mathrm{M}_{\odot}$ (e.g. Bromm \& Larson 2004). The supernovae from such stars would provide elemental abundance patterns in the stars they enrich that do not match those of the extremely metal-poor stars. An ELT could perhaps see massive Population III starbursts at high redshift.

- and the dark matter - how is this related, and is its physics really trivially simple??

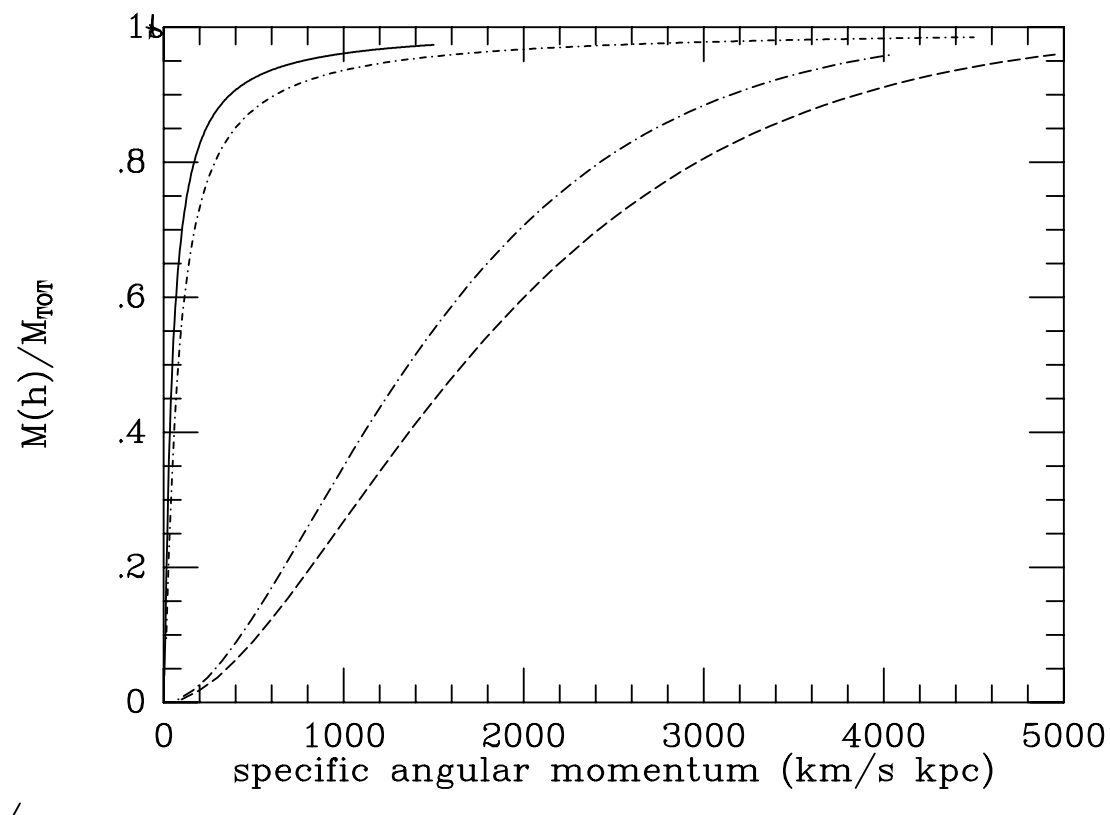

Figure 2. Adapted from Wyse \& Gilmore 1992, their Figure 1. Angular momentum distributions of the bulge (solid curve), the stellar halo (short-dashed/dotted curve), the thick disk (long-dashed/dotted curve) and the thin disk (long-dashed curve). The bulge and stellar halo have similar distributions, as do the thick and thin disks. Does this hold for external galaxies, pointing to fundamental relationships between bulge and halo, and thick and thin disks? An ELT with an IFU could tell us. 


\section{Implications for ELT capabilities}

It is not our purpose here to quantify a science requirements document for any specific telescope. Rather, we end by noting that the wide range of science questions briefly introduced above require observations beyond the Local Group, and observations with high sensitivity and high spatial resolution. It is a reasonable assumption that extant 8-10m telescopes will be developed and used to their limits, with the same set of nextgeneration enhancements that are also the learning curve for the ELT-instruments and capabilities.

International technological developments, using the best 8-10m telescopes as test-beds, will both optimise scientific gains for today's astronomy, and allow development of next generation facilities. The science questions introduced above are those we believe will survive our best efforts over the next decade, and truly need next generation capabilities.

Extending current efforts to the ELTs can push galaxy formation understanding into contact with reality, with testable predictions, and real science as ambitious outcomes.

\section{References}

Abadi, M., Navarro, J., Steinmetz, M. \& Eke, V. 2003, ApJ 597, 21

Aoki, W. et al. 2005, ApJ in press (astro-ph/0509206)

Beers, T. et al. 2000, $A J$ 119, 2866

Bell, E. \& de Jong, R. 2001, ApJ 550, 212

Bell, E. et al. 2005, ApJ 625, 23

Bensby, T., Feltzing, S. \& Lundström, I. 2004, A\&A 415, 155

Binney, J., Dehnen, W. \& Bertelli, G. 2000, MNRAS 318, 658

Bernardi, M. et al. 2003, AJ 1251817

Bernardi, M. et al. 2005, AJ 12961

Boylan-Kolchin, M., Ma, C-P. \& Quataert, E. 2005, MNRAS 362, 184

Bromm, V. \& Larson, R. 2004, ARAA 42, 79

Brown, T.M et al. 2003, ApJ 592, L17

Brown, T.M et al. 2005, astro-ph/0512001

Bullock, J., Kravtsov, A. \& Weinberg, D. 2000, ApJ 539, 517

Cimatti, A. et al. 2005, Nature 430, 184

Cooray, A. \& Cen, R. 2005, ApJ 63369

Cole, S., Lacey, C., Baugh, C. \& Frenk, C. 2000, MNRAS 319, 168

Croton, D.J. et al. 2006, MNRAS 365, 11

Cowie, L., Songaila, A., Hu, E. \& Cohen, J. 1996, AJ 112, 839

Dekel, A. \& Woo, J. 2003, MNRAS 344, 1131

Dekel, A. \& Silk 1986, ApJ 303, 39

De Simone, R., Wu, X. \& Tremaine, S. 2004, MNRAS 350, 627

Djorgovski, S. \& Davis, M. 1987, ApJ 313, 59

Dressler. A. et al. 1987, ApJ 313, 42

Efstathiou, G. 1992, MNRAS 256, 43

Eke, V., Efstathiou, G. \& Wright, L. 2000, MNRAS 315, L18

Fall, S.M. \& Efstathiou, G. 1980, MNRAS 193, 189

Famaey, B. et al. 2005, A\&A 430, 165

Ferguson, A.M.N., Irwin, M., Ibata, R., Lewis, G \& Tanvir, N. 2002, AJ 124, 1452

Ferguson, A.M.N. et al. 2005, ApJ 622, L109

Ferguson, A.M.N., Irwin, M, Chapman, S., Ibata, R., Lewis, G. \& Tanvir, N. 2006, in: R. de Jong (ed.), 'Island Universes - Structure \& Evolution of Disk Galaxies', Springer (Dordrecht), in press

Ferrarese, L. \& Merritt, D. 2000, ApJ 539, L9

Gebhardt, K. et al. 2000, ApJ 539, L13

Gilmore, G. \& Reid, I.N. 1983, MNRAS 202, 1025 
Glazebrook, K. et al. 2005, Nature 430, 180

Heavens, A., Panter, B., Jimenez, R. \& Dunlop, J. 2004, Nature 428, 625

Ibata, R., Gilmore, G. \& Irwin, M. 1994, Nature 370, 194

Ibata, R., Irwin, M., Lewis, G., Ferguson, A. \& Tanvir, N. 2001, Nature 412, 49

Ibata, R., Irwin, M., Lewis, G., Ferguson, A. \& Tanvir, N. 2003, MNRAS 340, L21

Jimenez, R., Panter, B., Heavens A.F. \& Verde L. 2005, MNRAS 356, 495

Kauffmann, G. 1996, MNRAS 281, 487

Kauffmann, G. \& Charlot, S. 1998, MNRAS 294, 705

Kauffmann, G. \& Haenelt, M. 2000, MNRAS 311, 576

Klypin, A. et al. 1999, ApJ 522, 82

Larson, R. \& Tinsley, B. 1979, MNRAS 186, 503

Makino, J. \& Hut, P. 1997, ApJ 481, 83

Mateo, M. 1998, ARAA 36, 435

Mo, H., Mao, S. \& White, S.D.M. 1998, MNRAS, 295319

Moore, B. et al. 1999, ApJ 524, L19

Mould, J. 2005, AJ 129, 698

Nagashima, M. et al. 2005, MNRAS 363, L31

Navarro, J. \& Steinmetz, M. 1997, ApJ 478, 13

Newberg, H. et al. 2002, ApJ 569, 245

Ostriker, J.P. 1990, in: R.G. Kron (ed.), 'Evolution of the Universe of Galaxies', ASP Conference proceedings volume $10, \mathrm{p} 25$

Robertson, B., Bullock, J., Font, A., Johnston, K. \& Hernquist, L. 2005, ApJ 632, 872

Saito, M. 1979, PASJ 31, 193

Sandage, A. 1965, in: H. Bondi (ed.), 'The Structure and Evolution of Galaxies', Interscience (New York) p83

Silk, J. \& Rees, M.J. 1998, A\&A 331, L1

Springel, V. et al. (Millenium Simulation) 2005, Nature 435, 629

Tran, K-V. et al. 2005, ApJL 627, L25

Treu, T. et al. 2005, ApJ 633, 174

Tremonti, C. et al. 2004, ApJ 613898

Trujillo, I. et al. 2006, submitted to $A p J$, astro-ph/0504225

Unavane, M., Wyse, R.F.G. \& Gilmore, G. 1996, MNRAS 278, 727

van Dokkum, P., Franx, M., Kelson, D. \& Illingworth, G. 1998, ApJL 504, L17

van Dokkum, P. 2005, AJ 130, 2647

Wyse, R.F.G. \& Gilmore, G. 1992, AJ 104, 144

Wyse, R.F.G. \& Silk, J. 1985, ApJL 296, L1

Yoachim, P. \& Dalcanton, J. 2005, ApJ 624, 701

Zurek, W., Quinn, P. \& Salmon, J. 1988, ApJ 330, 519 